\title{
Tratamento endovascular das obstruções venosas crônicas do segmento iliocaval
}

\author{
Endovascular treatment of chronic obstructions of the iliocaval segment \\ Giuliano de Almeida Sandri
}

\begin{abstract}
Resumo
A insuficiência venosa crônica é um grave problema de saúde pública no mundo, consumindo grandes quantias de recursos e causando grande prejuízo na qualidade de vida dos pacientes portadores de suas formas mais avançadas. A cirurgia para o tratamento de obstruções no sistema venoso profundo não foi incorporada à prática da maioria dos cirurgiões vasculares, ficando restrita a poucos centros em alguns países. Com o advento da cirurgia endovascular, a possibilidade de tratar alguns tipos de lesões obstrutivas por uma técnica minimamente invasiva e com resultados promissores renova o interesse da comunidade vascular pelas formas mais complexas de doença venosa.
\end{abstract}

Palavras-chave: Insuficiência venosa; síndrome pós-trombótica; angioplastia.

\begin{abstract}
Chronic venous insufficiency is an important public health issue worldwide, that consumes significant amounts of resources and impairs the quality of life of patients who suffer from its more severe clinical types. Surgery for the treatment of deep venous system obstruction has not been incorporated to the practice of most vascular surgeons, being restricted to a few medical centers in some countries. With the advent of endovascular surgical techniques, the possibility of treating some obstructive lesions with a minimally invasive technique that has promising results has renewed the interest of the vascular community for the treatment of more complex forms of vascular disease.
\end{abstract}

Keywords: venous insufficiency; post-thrombotic syndrome; angioplasty.

\section{Introdução}

A insuficiência venosa crônica (IVC) é um problema muito prevalente na sociedade moderna e, quando se considera todo seu quadro clínico, algum grau atinge até $85 \%$ dos indivíduos estudados ${ }^{1}$. Em 2004, realizou-se um consenso que publicou a versão atual do sistema de classificação CEAP para a IVC, fornecendo padronização internacional compreensiva e completa de diferentes critérios, para fins tanto clínicos quanto científicos².

A classificação CEAP ganhou cada vez mais aceitação nos últimos anos, porém ainda é subutilizada, sobretudo para fins científicos. Existe grande dificuldade de se obterem trabalhos epidemiológicos sobre a verdadeira prevalência da doença venosa na população geral, o que é reflexo da pouca importância atribuída à IVC, principalmente por não cursar com risco ao membro ou à vida do paciente, em contraposição a outras doenças vasculares, como a doença arterial obstrutiva periférica (DAOP) com isquemia crítica. Apesar da percepção de que a IVC possui evolução benigna, seus espectros mais avançados (edema e alterações cutâneas incluindo úlceras) são causas de grande transtorno psicológico e social aos seus portadores, principalmente porque têm importante impacto na qualidade de vida dos mesmos, além de gerar enorme quantidade de custos relacionados ao absenteísmo no trabalho ou às complicações clínicas que recebem acompanhamento em serviços de saúde, o que pode consumir de 1 a 3\% dos recursos orçamentários destinados a saúde em países desenvolvidos ${ }^{1,3}$. Estudo publicado sobre IVC no Brasil analisou a prevalência de sinais da doença avançada relacionada a varizes e encontrou uma média de 19,7\% para edema, 5,7\% para hiperpigmentação, 1,4\% para eczema e $0,6 \%$ para dermatofibrose ${ }^{4}$.

'Médico Assistente do Serviço de Cirurgia Vascular do Hospital do Servidor Público Estadual "Francisco Morato de Oliveira" (HSPE), São Paulo (SP), Brasil.

Não foram declarados conflitos de interesse associados à publicação deste artigo.

Submetido em: 13.10.2010. Aceito em: 28.05.2011

J Vasc Bras. 2011;10(2):137-144. 
Além dos sinais observados, pacientes portadores de IVC apresentam sintomatologia variada e, às vezes, de difícil atribuição a doença venosa exclusivamente, sobretudo nos casos menos avançados (categorias C0 a C2 da classificação CEAP $)^{5,6}$. Existe, no entanto, piora importante da sintomatologia de acordo com a progressão da classe clínica ${ }^{3,7,8}$, e o impacto na qualidade de vida dos pacientes portadores de IVC avançada (classes C5 e C6) pode ser semelhante ao causado pela insuficiência cardíaca ${ }^{1}$. Os sintomas são geralmente associados a desconfortos sensitivos, principalmente sensação de cansaço e dor. Cãibras são frequentes, porém muito inespecíficas, e o prurido pode se manifestar em fases iniciais, mas está geralmente associado ao eczema. A claudicação venosa, caracterizada por dor de forte intensidade com o exercício físico intenso, é sintoma raro e ocorre em casos mais avançados de doença venosa ${ }^{4,8}$.

Os mecanismos fisiopatogênicos responsáveis pelas alterações observadas na IVC ainda não estão completamente elucidados, porém as manifestações clínicas parecem estar relacionadas com dois mecanismos hemodinâmicos básicos: refluxo valvular e obstrução na via de efluxo ou escoamento ${ }^{1,5-11}$. Alguns pesquisadores sugerem que a obstrução associa-se mais à dor e ao edema nos membros inferiores, enquanto o refluxo está ligado às alterações cutâneas, inclusive às ulcerações ${ }^{8}$. Observa-se com frequência a presença de ambos os mecanismos fisiopatogênicos na maioria dos indivíduos portadores de doença venosa moderada a avançada ${ }^{3,8,11,12}$.

Além do tratamento clínico, o tratamento cirúrgico, com todas as suas variações, tornou-se o padrão-ouro na correção do refluxo valvular do sistema venoso superficial e perfurante dos membros inferiores através de métodos de interrupção, tais como a safenectomia convencional e a ligadura subfascial de perfurantes videoassistida ${ }^{6}$. Já o tratamento cirúrgico do refluxo no sistema profundo e da doença obstrutiva do sistema venoso, presentes nos casos mais avançados de IVC, apresentam formas de tratamento mais complexas e com resultados menos satisfatórios, o que levou à não difusão dos métodos para a prática dos cirurgiões vasculares, com exceção daqueles poucos que trabalham em centros de referência em doença venosa ${ }^{13-15}$.

Com os avanços adquiridos na área de angiorradiologia e cirurgia endovascular nos últimos anos, despertou-se um novo interesse pelo tratamento das obstruções venosas do segmento iliofemoral e da veia cava inferior, surgindo a possibilidade de se oferecer uma melhora importante na qualidade de vida dos indivíduos portadores desta doença por meio de procedimentos minimamente invasivos.

Embora o termo "oclusão" e "obstrução" sejam utilizados na Medicina como designação de fechamento luminal completo de uma estrutura, a acepção correta permite que esses termos sejam empregados para qualquer tipo de diminuição da luz, seja parcial ou total ${ }^{16}$. A literatura internacional utiliza esses termos de maneira confusa, obedecendo a um ou outro conceito e, portanto, o presente manuscrito apresenta os termos parcial ou total quando para se referir às lesões, evitando a falta de clareza semântica.

A obstrução venosa do segmento iliocaval pode ser caracterizada como de etiologia trombótica ou não, mesmo na fase crônica. Quando as obstruções totais e parciais são levadas em conta nos pacientes sintomáticos, existe uma predominância das lesões não trombóticas (até 53\% dos pacientes), principalmente compressões extrínsecas descritas, como a síndrome de Cockett, que alguns autores denominam atualmente de "lesões obstrutivas sintomáticas não trombóticas das veias ilíacas", mais prevalentes em mulheres e no lado esquerdo. Quando somente as oclusões totais não relacionadas a compressões tumorais são avaliadas (foco deste manuscrito), quase todos os casos apresentam etiologia pós-trombótica, associada ou não às compressões extrínsecas ${ }^{5,15,17-20}$.

Em 1958, Palma descreveu a cirurgia para tratar obstrução venosa ilíaca unilateral, utilizando uma derivação fêmoro-femoral cruzada com veia safena magna contralateral. Com o desenvolvimento das próteses, realizaram-se também derivações axiais (isto é, fêmorocaval) e cruzadas com ePTFE (expanded polytetrafluoroethylene). Além disso, o enxerto de veia safena em espiral também foi descrito para as obstruções proximais. Esses tipos de cirurgias são realizados por poucos grupos e em um número pequeno de pacientes, o que torna a análise científica dos resultados passível de muitas críticas. No entanto, perviedades secundárias de até $83 \%$ para o enxerto com safena (cirurgia de Palma) e de $53 \%$ para o ePTFE axial foram descritos, com uma melhora clínica sempre satisfatória ${ }^{13,14}$.

A angioplastia transluminal percutânea foi utilizada pela primeira vez como alternativa terapêutica para as obstruções venosas em casos de síndrome de Budd-Chiari e lesões das veias hepáticas, porém o resultado tinha duração temporária e necessitava de várias reintervenções. Os primeiros stents utilizados no sistema venoso datam de 1969 e foram descritos por Cesar Gianturco et al.; o primeiro stent implantado na veia cava inferior foi relatado em 1986 por Zollikofer et al. No início, alta incidência de reestenose por hiperplasia miointimal e de migração eram observadas, mas houve diminuição considerável desses problemas com a evolução da tecnologia dos materiais e da técnica endovascular ${ }^{18}$. 


\section{Avaliação pré-operatória e preparo para intervenção}

Os pacientes candidatos à terapia de recanalização endovascular do território venoso cavo-ilíaco-femoral devem ser submetidos a uma avaliação pré-operatória sistemática. O diagnóstico geralmente é sugerido pelo quadro clínico e por exames não invasivos, como o duplex scan venoso, pletismografia a ar, diferença de pressão venosa entre braços e pernas e mensuração de pressão venosa ambulatorial, porém esses exames não possuem especificidade e sensibilidade adequadas para a detecção e quantificação da obstrução venosa $a^{11,18,19}$. O diagnóstico definitivo é determinado por métodos de imagem, como a flebografia ascendente e a flebografia por punção de veia femoral, principalmente pela última, que demonstra mais adequadamente o território proximal e permite a identificação de diversas alterações da doença venosa obstrutiva, tais como oclusões parciais e presença de colaterais pélvicas no segmento acometido (Figura 1). A angiotomografia e a angiorressonância magnética apresentam imagens e capacidade diagnóstica semelhantes à flebografia convencional, porém são menos utilizadas para estudar o território venoso ${ }^{8}$. A maioria dos

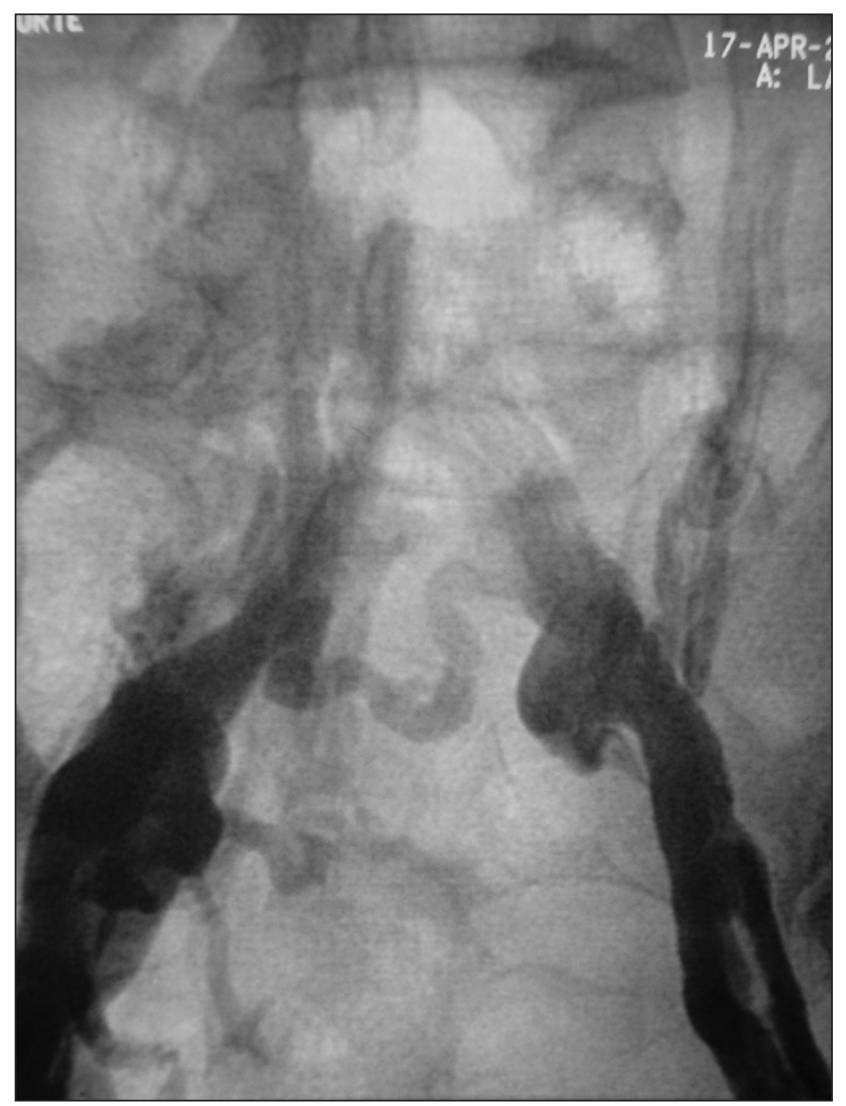

Fonte: imagem cedida pelo Dr. João Luiz Sandri.

Figura 1 - Angiografia da oclusão crônica do território ilíaco-cavo com atenção especial para a extensa circulação colateral pélvica. pacientes já está em tratamento clínico otimizado, com melhora insatisfatória ${ }^{15,18-22}$.

Além dos exames pré-operatórios de rotina, os candidatosao tratamento devem ser investigados para as trombofilias hereditárias ou adquiridas mais comuns. Todas as informações clínicas, tais como a classificação da doença e quantificação da severidade, devem ser bem registradas. Medidas da circunferência e fotografias dos membros inferiores e também devem ser devidamente arquivadas ${ }^{18,22}$. A aplicação de questionários específicos para IVC também é de grande utilidade, principalmente para avaliar a melhora da qualidade de vida após intervenção ${ }^{19}$. O uso de fibrinolítico deve ser considerado na avalição pré-operatória e necessita de certos cuidados adicionais, como a dosagem de fibrinogênio, a reserva de hemoderivados e pós-operatório em unidade de cuidados intensivos ${ }^{18}$.

Os pacientes devem ser plenamente esclarecidos sobre os aspectos técnicos do procedimento, suas potenciais complicações e quais serão as melhoras esperadas. Após o procedimento, há necessidade de seguimento contínuo e por tempo indeterminado e, portanto, um grande compromisso deve ser assumido entre o paciente e a equipe para que o sucesso do tratamento seja durável ${ }^{18-20}$.

\section{Técnica cirúrgica}

O procedimento de recanalização do território iliocaval deve ser tratado como uma cirurgia de grande porte e todos os cuidados necessários devem ser tomados ${ }^{18}$. Os pacientes que estão em uso de anticoagulante oral devem ter a medicação suspensa, de acordo com a rotina do serviço. A anestesia pode ser local com sedação ou até mesmo geral, por se tratar de um procedimento potencialmente demorado e que gera muita dor e desconforto para o paciente ${ }^{18-22}$.

A escolha dos locais de acesso a serem utilizados é de suma importância e deve ser feito de maneira criteriosa. Para assegurar a perviedade do tratamento, o segmento recanalizado deve ser encarado como segmento arterial doente, que deverá ser tratado com uma derivação e, portanto, deve possuir um bom influxo e escoamento para que não ocorra trombose precoce. $\mathrm{O}$ acesso deve possibilitar que toda área doente seja tratada, garantindo espaço para liberação de stents e insuflação de balões além da bainha introdutora $^{18,19}$.

A via de acesso preferencial é a veia femoral comum e geralmente existe necessidade de mais de um local de acesso (por exemplo, punção da veia femoral comum bilateralmente). Quando a doença acomete essa veia, o que não é raro, ela também precisará ser tratada e, portanto, o acesso terá que ser feito por outras vias. As alternativas mais utilizadas 
são as veias femorais (antigamente chamadas de veias femorais superficiais), as veias poplíteas (que exigem decúbito ventral do paciente) e a veia jugular interna direita ${ }^{18,19,22,23}$. Opções menos comums são as veias femorais profundas e tributárias vicariantes da região femoral ${ }^{19}$. O uso de punção guiada por ultrassom diminui muito a ocorrência de complicações e já é rotina na maioria dos serviços ${ }^{15,18-20,23}$. Em casos extremos, pode-se se fazer um acesso cirúrgico com trombectomia e endoflebectomia da veia femoral comum para passagem dos dispositivos ${ }^{18,19,22}$.

O acesso é realizado por técnica de Seldinger e passagem de bainha introdutora de 5 ou 6 Fr de $15 \mathrm{~cm}$, com utilização de fluoroscopia e subtração digital. Para atravessar a lesão, um fio-guia hidrofílico de 0,035 ”, com a ponta flexível e um cateter com curva de ângulo reto, é a escolha inicial. O processo de atravessar toda a área doente é demorado e tedioso. Durante o procedimento, utiliza-se heparina sistêmica para anticoagulação ${ }^{15,18-23}$.

Quando existe grande dificuldade de avanço do conjunto, não se deve forçá-lo, devido ao risco de perfuração. Uma opção é trocar o fio guia mais fino e tentar dilatar o trajeto com um balão pequeno (de 2 a $4 \mathrm{~mm}$ de diâmetro) antes de avançar o conjunto ${ }^{18}$, embora alguns autores considerem essa manobra contraproducente ${ }^{19}$. Isso também pode ser feito nos casos em que o fio-guia já atravessou toda a região acometida, mas o cateter não progride. A troca por fios-guia rígidos de suporte deve sempre ser feita de maneira protegida (por dentro de um cateter) como em outros procedimentos endovasculares. A técnica do "varal" pode ser utilizada para gerar mais sustentação, mas necessita obrigatoriamente de um acesso pela veia jugular interna ${ }^{18,19}$. Alguns autores defendem o uso de fibrinolítico local com intuito de "amolecer" parte do trombo antigo ou dissolver um trombo mais recente no meio deste e abrir uma pequena passagem que possa permitir o avanço do fio-guia. A infusão pode ser feita em bôlus, com a tentativa de prosseguir após alguns minutos, ou pode ser feita de maneira lenta e contínua na UTI, com interrupção do procedimento e continuação no dia seguinte ${ }^{18}$.

$\mathrm{O}$ avanço do fio-guia e do cateter deve ser feito sempre com cuidado, e a posição deve ser verificada com diferentes incidências, se necessário. Algumas áreas, como a confluência das veias ilíacas comuns, requerem cuidados maiores porque nelas existem várias veias tributárias que seguem paralelas à veia cava inferior, e o fio-guia pode estar em um falso trajeto por umas dessas veias. Após posicionar o conjunto em uma posição livre de doença (de acordo com a flebografia realizada), realiza-se uma nova aquisição confirmatória ${ }^{18,19}$.
O uso de ultrassom endovascular (IVUS, do inglês intravascular ultrasound) é considerado parte fundamental do procedimento, sendo utilizado rotineiramente por Raju e Néglen. Esses autores defendem que com o IVUS é possível dilatar a veia doente para o tamanho correto em um único passo, sem risco de rotura por utilização de um cateter balão maior que o necessário. Além disso, lesões menores e que podem passar despercebidas pela flebografia digital são detectadas pelo IVUS e, assim, toda área doente pode ser tratada, sem prejuízo à função final da recanalização ${ }^{19}$. O IVUS, no entanto, é muito caro e muitos autores não dispõem da tecnologia ou a utilizam em casos selecionados ${ }^{18,23}$. Os diâmetros dos balões utilizados variam de 10 a $24 \mathrm{~mm}$, de acordo com o segmento.

Os stents utilizados devem ser autoexpansíveis e exceder o diâmetro estimado do vaso em 2 a $4 \mathrm{~mm}^{19}$. Existe uma preferência pelo uso do Wallstent (Boston Scientific, Natick, MA, USA), porém os stents de nitinol também podem ser utilizados. O IVUS também facilita a escolha do diâmetro e comprimento do stent ${ }^{19}$, porém algumas manobras, como a insuflação de um balão até um diâmetro conhecido com tração do mesmo dentro da veia, podem ajudar, já que, se o mesmo se deslocar com facilidade, um stent pelo menos $20 \%$ maior que o diâmetro do balão será utilizado ${ }^{18,19}$. Toda área doente deve ser recoberta, inclusive a veia femoral comum quando acometida. A fratura de stents que cruzam o ligamento inguinal no território venoso é descrita como complicação rara ${ }^{18,19,22-24}$. A liberação dos stents é feita de maneira proximal para distal e podemse utilizar diâmetros diferentes para segmentos diferentes (por exemplo, $12 \mathrm{~mm}$ para a veia femoral comum e ilíaca externa, 14 a $16 \mathrm{~mm}$ para veia ilíaca comum e 18 a $20 \mathrm{~mm}$ para veia cava inferior $)^{18}$ ou utilizar diâmetro único para todos os segmentos, com a escolha do stent recaindo sobre o maior diâmetro a ser utilizado. Este último método permite que expansão do stent e, portanto, seu diâmetro final, seja limitado pelo diâmetro luminal dos diferentes segmentos tratados ${ }^{19}$.

A sobreposição entre cada stent consecutivo deve ser de, pelo menos, $2 \mathrm{~cm}^{19,22}$ para evitar migrações e desconexões, e os mesmos devem ter o maior comprimento possível. Quando é necessário liberar um stent na veia cava inferior por doença ilíaca unilateral, não parece ser necessário fazer a técnica dos kissing stents como no sistema arterial, uma vez que o fluxo contralateral atravessa bem pela malha do stent e não há aumento de tromboses significativas ${ }^{18,19,22}$. Quando existe doença ilíaca bilateral, diversas configurações de stents são descritas, tais como stents saindo de cada veia ilíaca e permanecendo paralelos na veia cava inferior 


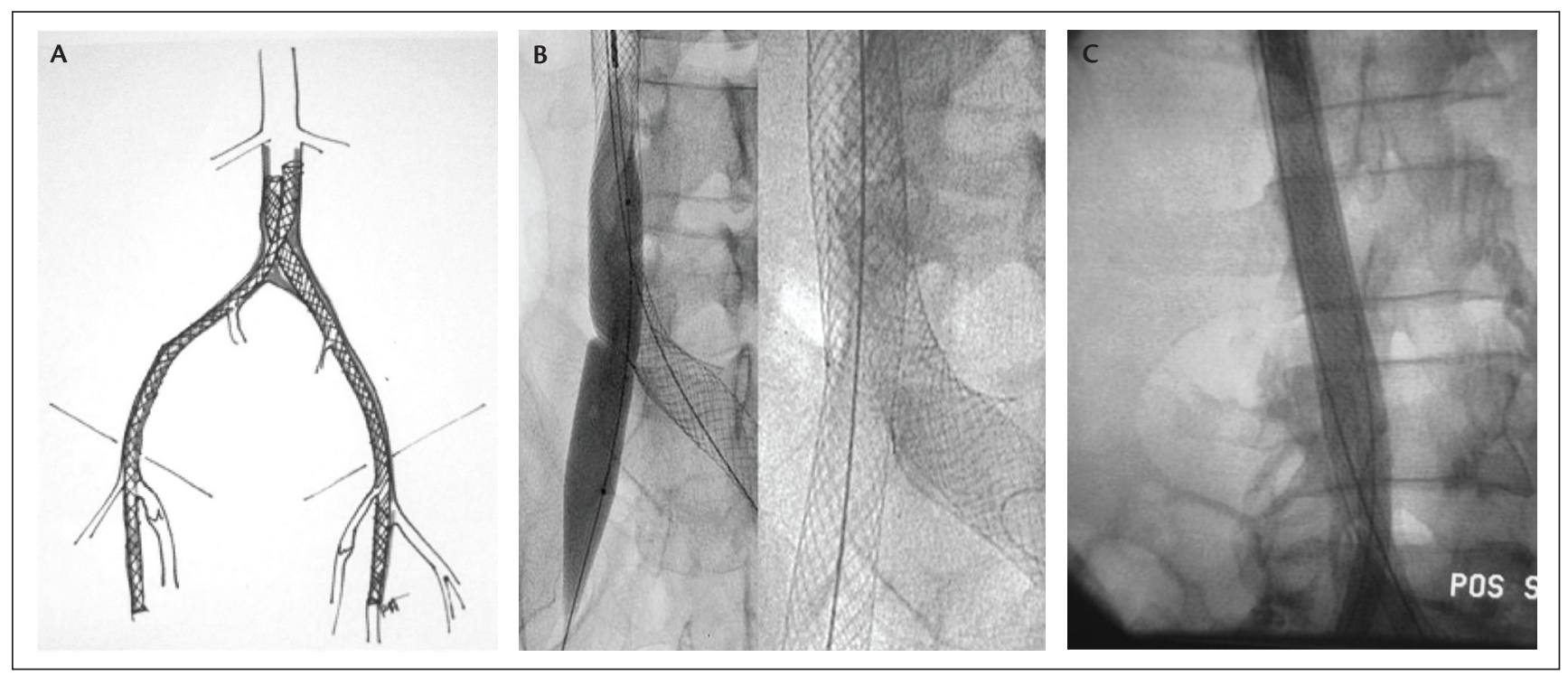

Fonte: imagem cedida pelo Dr. João Luiz Sandri.

Figura 2 - Exemplos de configuração dos stents na veia cava inferior e confluência das veias ilíacas comuns. (A) Ilustração por stents paralelos ou kissing stents (Thorpe e Osse ${ }^{18}$ ). (B) "Y" invertido, em que o stent mais curto é atravessado e dilatado pela malha metálica do stent já implantado (Raju e Neglén ${ }^{19}$ ). (C) "Y" invertido modificado, com dois stents ilíacos paralelos por dentro de um stent maior na veia cava inferior.

até acima da área doente (Figura 2A) ${ }^{18,23}$ em "Y" invertido, em que um dos stents ilíacos continua pela veia cava inferior até o nível sem doença e o contralateral termina distalmente através de uma fenestração feita na malha do stent mais longo ${ }^{19}$ (Figura 2B) ou ainda em "Y" invertido modificado em que os stents das ilíacas terminam dentro de um stent único na cava inferior (Figura $2 \mathrm{C})^{18}$. Após a liberação de todos os stents, faz-se a dilatação com balão para o tamanho adequado de cada segmento.

A presença de filtro de veia cava inferior no segmento a ser tratado não constitui uma contraindicação ao procedimento, embora torne o procedimento mais desafiador. Nesses casos, o fio-guia deve passar entre as hastes de metal do filtro e não por fora dele, para que a dilatação com balão se desfaça e abra a estrutura do mesmo, de maneira que os stents se expandam sem dificuldade ${ }^{18,19}$.

O controle final é feito com uma injeção distal e é considerado adequado quando se observa passagem rápida de contraste, sem retenção do mesmo no interior da segmento tratado e com desaparecimento ou redução importante do enchimento da circulação colateral pélvica, mostrando que o fluxo está preferencialmente no conduto recém criado ${ }^{15,18-}$ ${ }^{23}$. O uso de IVUS pode ainda detectar lesões imperceptíveis à flebografia digital ${ }^{19}$.

As bainhas são removidas em vigência de anticoagulação e o paciente fica em repouso por 24 horas. A grande maioria dos autores inicia varfarina ou outro anticoagulante oral no pós-operatório, mantendo a heparina não fracionada até atingir relação normatizada internacional (RNI) terapêutico (entre 2,0 e 3,0). O anticoagulante oral é mantido por pelo menos seis meses, associado ou não ao clopidogrel ${ }^{18,22,23,25}$. Alguns serviços preconizam apenas o uso de antiagregante plaquetário no pós-operatório, exceto para aqueles pacientes que possuem indicação de anticoagulação pela doença de base ${ }^{19}$. Como a maioria dos pacientes com oclusões crônicas totais de território iliocaval são portadores de síndrome pós-trombótica, a indicação de anticoagulação oral se impõe.

O seguimento é feito com retornos ambulatoriais de rotina para exame clínico e pelo menos um exame de duplex scan anual ou com menor intervalo, pelo menos no primeiro ano. Se sinais ou sintomas como dor, desconforto e edema recorrerem ou alterações no ultrassom forem detectadas, a manutenção da perviedade pode ser feita com angioplastia simples via jugular interna direita, sem necessidade de internação ${ }^{18,22}$.

\section{Discussão}

A maioria dos trabalhos sobre o tratamento endovascular do território fêmoro-iliocaval analisaram conjuntamente os casos de obstrução parcial e total e fazem pouca menção específica aos casos de oclusão total. Raju e Néglen publicaram recentemente seus resultados no tratamento da oclusão tota ${ }^{19}$. Numa experiência de 1.402 membros tratados por doença venosa de 1999 a 2007, 167 (159 pacientes) 
pertenciam ao grupo da oclusão crônica total secundária a trombose venosa profunda (TVP). Todos os paciente eram sintomáticos e 32 tinham úlcera ativa. O sucesso técnico inicial foi de $72 \%$, porém, após insistência com novas tentativas (novos procedimentos realizados em dias diferentes), atingiu-se $83 \%$ de sucesso técnico. Quase metade dos stents distais foram liberados na veia femoral comum, cruzando o ligamento inguinal (47\%), e a grande maioria dos stents proximais foram implantados na veia cava inferior (98\%). As perviedades primária, primária assistida e secundária foram, respectivamente, de 31, 57 e 66\% em 48 meses, com erro padrão aceitável (Gráfico 1). A melhora clínica foi estatisticamente significativa para variáveis analisadas com o Chronic Venous Insufficiency Questionnaire (CVIQ), e não houve melhora ou piora nas variáveis hemodinâmicas avaliadas no estudo. A melhora da dor manteve-se em, aproximadamente, $80 \%$ em 4 anos (70\% de melhora completa); já o edema manteve melhora parcial e completa em, aproximadamente, 60 e $35 \%$ dos pacientes em 4 anos. Cerca de $58 \%$ dos pacientes tinham suas úlceras cicatrizadas e sem recidiva em 30 meses. Não se observaram fatores preditores de falha do tratamento neste estudo.

Razavi publicou casuística de tratamento de 17 oclusões totais, com sucesso técnico inicial de $88 \%$ e perviedade primária de $80 \%$ e primária assistida de $88 \%$ em 19 meses, porém sem análise dos resultados em relação a sintomatologia dos pacientes ${ }^{23}$. O grupo de Hartung, analisando casuística mista, relatou $93 \%$ de sucesso técnico para o tratamento das oclusões totais, porém não mencionou a perviedade específica para esse grupo de pacientes. Todos os 6 casos de CEAP C6 do estudo cicatrizaram, e houve melhora importante da

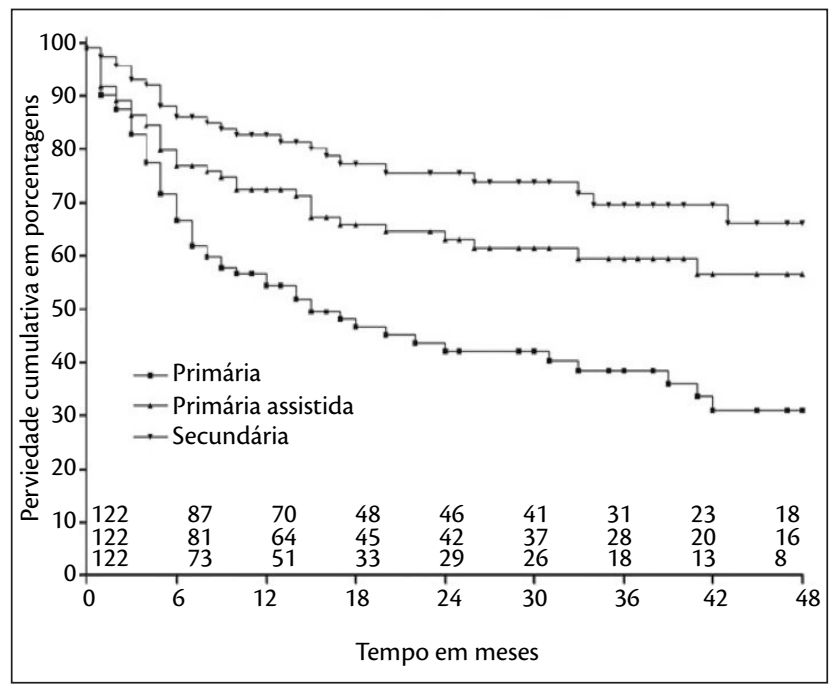

Fonte: Raju e Néglen ${ }^{19}$.

Gráfico 1 - Curva com estimativa de pervidades primária, primárias assistida e secundária. claudicação venosa ( $68 \%$ de cura e $27 \%$ de alívio). O acometimento da veia femoral comum foi um fator de risco para diminuição da perviedade primária, porém só manteve significância estatística na análise univariada ${ }^{22}$.

Não houve mortalidade relacionada ao procedimento nos diversos trabalhos analisados, e a incidência de complicações é baixa, sendo que a complicação mais frequentemente relatada foi a dor, controlada por analgesia simples. O hematoma no local de punção é raro quando comparado ao acesso arterial. Perfuração da cava inferior com hematoma retroperitoneal responde bem ao tratamento conservador. Elevação transitória dos níveis de creatinina, lesão iatrogênica da artéria femoral e infecção do stent também foram descritas e parecem ser muito raras. Porém, a trombose precoce ( $<30$ dias) é uma complicação presente na maioria das publicações ${ }^{15,18-23}$.

A cirurgia convencional para a doença venosa obstrutiva do território iliocaval está restrita a centros de referência, devido às limitações técnicas e clínicas. Grupos de excelência, como o da clínica Mayo, publicaram resultados satisfatórios com o tratamento cirúrgico, com nenhum óbito relacionado ao procedimento, baixos índices de complicações e perviedade secundária de $83 \%$ para a cirurgia de Palma em 4 anos e 54\% para as derivações axiais com PTFE em 2 anos $^{13}$. Em contrapartida, a terapia endovascular parece oferecer resultados promissores quando comparados ao tratamento cirúrgico, além de ampliar o número de indicações ${ }^{18}$.

Independentemente da técnica utilizada, os resultados são animadores, com melhoras na sintomatologia e na qualidade de vida de pacientes que geralmente apresentam expectativa de vida normal em relação aos indivíduos da mesma idade, diferentemente dos pacientes com doença arterial obstrutiva periférica com isquemia crítica. Porém, a doença venosa obstrutiva proximal carece ainda de métodos diagnósticos simples e não invasivos, hemodinâmicos ou de imagem, que permitam uma adequada avaliação pré-operatória da doença. Mesmo o segmento com duplex scan no pós-operatório dispõe de critérios que são análogos aos utilizados no sistema arterial, o que provavelmente é inadequado.

Os stents são utilizados liberalmente, atravessando o ligamento inguinal, e o número de fraturas não parece ser significativo, provavelmente porque o material sofre menos estresse do que o ambiente de alta pressão e com fluxo pulsátil do sistema arterial ${ }^{19,20,23,24}$. Isso implica numa escolha mais cuidadosa dos acessos, que podem ser realizados com complicações mínimas em locais pouco utilizados para os acessos arteriais (veia femoral na coxa e veia poplítea) ${ }^{18,19}$. Existe também uma preocupação em relação ao implante 
de stents na cava inferior em nível acima das veias renais, com possível prejuízo à função renal, uma vez que oclusões desse segmento chegam até esse local com relativa frequência. No entanto, esse tipo de complicação não foi descrito ${ }^{20}$. O sistema venoso parece tolerar melhor a carga metálica utilizada do que os sistema arterial ${ }^{19}$.

Como a prevalência da doença venosa proximal principalmente a lesões obstrutivas não trombóticas - é maior em mulheres jovens em idade fértil, existe uma preocupação crescente com o comportamento dos stents no território pélvico e a gestação. Hartung avaliou 62 pacientes em idade fértil submetidas à angioplastia com stent de segmento ilíaco-cavo das quais sete entraram em gestação com stents pérvios. A única oclusão durante a gravidez ocorreu em uma paciente com stent balão expansível, que foi esmagado pelo útero crescente. As demais pacientes possuíam stent autoexpansível, receberam enoxaparina profilática e fizeram duplex scan de vigilância em vários períodos da gestação. Quatro pacientes tiveram o diagnóstico de compressão e repercussão hemodinâmica sobre o stent e passaram a receber enoxaparina terapêutica. Nenhuma das pacientes com stent autoexpansível apresentou trombose e regressão dos sintomas de compressão após o término da gestação. Apesar do pequeno número, o uso de stents autoexpansíveis parece ser seguro, porém devem ser utilizados criteriosamente em mulheres jovens que planejam ter filhos ${ }^{26}$.

Existe também uma preocupação com a piora do refluxo venoso para o membro inferior acometido pela IVC. Alguns autores acreditam que, nos pacientes com componente de refluxo nas veias do membro e obstrução proximal de escoamento, este último agiria como fator protetor contra o primeiro e, se tratado, o aumento da intensidade do refluxo poderia levar a uma piora clínica, sobretudo das manifestações cutâneas. Na prática, esse problema não foi observado e, mesmo quando houve aumento do refluxo mensurável, houve melhora clínica dos pacientes, que continuaram utilizando compressão elástica ${ }^{15,19,25}$. Alguns grupos procederam com tratamento do refluxo do sistema venoso superficial no mesmo procedimento da recanalização endovascular, tanto por via convencional (safenectomia por eversão, ligadura) como por via minimamente invasiva (com laser ou radiofrequência) ${ }^{22,27}$.

A incidência de reestenose foi estudada por Néglen e Raju e é bastante alta nos stents implantados no segmento ilíaco-cavo (77\% em 2 anos). Porém, a maioria de reestenoses fica abaixo de $20 \%$ ou entre 20 e $50 \%$ (não significativas) e ocorre principalmente nos 2 anos após a implantação. Os fatores de risco associados a reestenoses não significativas e significativas (> 50\%) foram TVP prévia, estados trombofílicos, extensão do segmento com stent $>13 \mathrm{~cm} \mathrm{e}$ presença de stent cruzando o ligamento inguinal ${ }^{28}$.

Não existe qualquer protocolo estabelecido sobre o uso de medicamentos para prevenir trombose após recanalização venosa endovascular, porém a maioria dos autores concorda que aqueles pacientes com oclusão pós-trombótica, o que constitui a maioria dos casos, devem ser mantidos com regime de anticoagulação plena e prolongada ${ }^{18,22,23,25}$.

\section{Conclusão}

O sistema venoso profundo é cada vez mais submetido a técnicas intervencionistas endovasculares, tanto nos casos agudos (como na fibrinólise da TVP), como nos casos crônicos (como nas angioplastias das lesões parciais ou totais do território fêmoro-ilíaco-caval.

Com a divulgação e aprendizado do método de recanalização venosa endovascular, relativamente mais simples que a cirurgia convencional, pacientes sintomáticos que antes contavam apenas com um tratamento clínico rigoroso para conseguir pequeno alívio poderão contar com a chance de obter melhora clínica importante e rápida, por meio de um procedimento minimamente invasivo, seguro e eficiente, com um índice baixo de complicações. Com o renascimento do interesse pelo estudo e pela terapia das doenças venosas, o método endovascular evolui e se difunde rapidamente e, quiçá, a cirurgia para os problemas venosos complexos passará também a ser realizada em centros onde antes era desconhecida ou foi abandonada.

\section{Referências}

1. Bergan J, Schmid-Schönbei G, Smith P, Nicolaides A, Boisseau M, Eklöf B. Mechanisms of desease: chronic venous desease - review article. N Engl J Med. 2006;355:489-498.

2. Eklöf B, Rutherford RB, Bergan Jj, Carpentier PH, Gloviczki P, Kistner RL, Meissner MH, Moneta GL, Myers K, Padberg FT, Perrin M, Ruckley CV, Smith PC, Wakefield TW; American Venous Forum International Ad Hoc Committee for Revision of the CEAP Classification. Revision of the CEAP classification for chronic venous desorders: consensus statement. J Vasc Surg. 2004;40:1248-52

3. Delis KT, Bountouroglou D, Mansfield AO. Venous claudication in iliofemoral thrombosis: long-term effects on venous hemodynamics, clinical status, and quality of life. Ann Surg. 2004;239:118-26.

4. Maffei FHA, Magaldi C, Pinho SZ, et al. Varicose veins and chronic venous insufficency in Brazil: prevalence among 1755 inhabitants of a country town. Int J Epidemiol 1986;15:210-7.

5. Santos M. Varizes dos membros inferiores. Conceito, Etiopatogenia, Quadro Clínico e Diagnóstico. In: Brito C], editor. Cirurgia Vascular. Rio de Janeiro: Revinter; 2008. p. 1497-529.

6. Gloviczki P. The management of venous disorders: introduction and general considerations. In: Rutherford RB, editor. Vascular Surgery. Philadelphia: Elsevier Saunders; 2005. p. 2111-23. 
7. Kaplan RM, Criqui M, Denenberg JO, Bergan J, Fronek A. Quality of life in patients with chronic venous disease: San Diego population study. J Vasc Surg. 2003;37:1047-53.

8. Néglen P. Importance, Etiology, and Diagnosis of Chronic Proximal Venous Outflow Obstruction. In: Bergan Jj, editor. The Vein Book. Burlington: Academic Press; 2007. p. 541-48.

9. Kahn SR, M'lan CE, Lamping DL, Kurz X, Bérard A, Abenhaim LA; VEINES Study Group. Relationship between clinical classification of chronic venous disease and patient-reported quality of life: results from an international cohort study. J Vasc Surg. 2004;39:823-8.

10. Killewich LA, Martin R, Cramer M, Beach KW, Strandness DE Jr. Pathophysiology of venous claudication. J Vasc Surg. 1984;1:507-11.

11. Labropoulos $\mathrm{N}$, Volteas $\mathrm{N}$, Leon $\mathrm{M}$, et al. The role of venous outflow obstruction in patients with chronic venous dysfunction. Arch Surg. 1997;132:46-51.

12. Néglen P, Thrasher TL, Raju S. Venous outflow obstruction: An underestimated contributor to chronic venous disease. I Vasc Surg. 2003;38:879-85

13. Jost CJ, Gloviczki P, Cherry KJ Jr, et al. Surgical reconstruction of iliofemoral veins and the inferior vena cava for nonmalignant occlusive disease. J Vasc Surg. 2001;33:320-8.

14. Néglen P. Treatment of iliac venous obstruction in chronic venous desease. In: Bergan, JJ. The Vein Book. Burlington: Academic Press 2007;549-57.

15. Mussa FF, Peden EK, Zhou W, Lin PH, Lumsden AB, Bush RL. Iliac vein stenting for chronic venous insufficiency. Tex Heart Inst J. 2007;34:60-6.

16. Houaiss A. Dicionário Houaiss da língua portuguesa. Rio de Janeiro: Objetiva; 2001.

17. Néglen P. Endovascular reconstruction for chronic iliofemoral vein obstruction. In: Gloviczki P, Yao JST. Handbook of venous disorders: guidelines of the American Venous Forum. Hachette: Hodder Education; 2009. p. 491-502.

18. Thorpe P, Osse FJ. Endovenous management of Iliocaval occlusion. In: Bergan JJ, editor. The Vein Book. Burlington: Academic Press; 2007. p. 559-74.
19. Raju S, Néglen P. Percutaneus recanalization of total occlusion of the iliac vein. J Vasc Surg. 2009;50:360-8.

20. Bjarnason $\mathrm{H}$. Endovascular reconstruction of complex iliocaval venous occlusions. In: Gloviczki P, Yao JST. Handbook of venous disorders: guidelines of the American Venous Forum. Hachette: Hodder Education; 2009. p. 503-13.

21. Hartung $O$, Otero A, Boufi $M$, et al. Mid-term results of endovascular treatment for symptomatic chronic nonmalignant iliocaval venous occlusive disease. J Vasc Surg. 2005;42:1138-44.

22. Hartung $O$, Loundou AD, Barthelemy P, Arnoux D, Boufi M, Alimi YS. Endovascular management of chronic disabling ilio-caval obstructive lesions: long term results. Eur J Vasc Endovasc Surg. 2009;38:118-24.

23. Razavi MK, Hansch EC, Kee ST, Sze DY, Semba CP, Dake MD. Chronically occluded inferior venae cavae: endovascular treatment. Radiology. 2000;214:133-8.

24. Neglén P, Tackett TP Jr, Raju S. Venous stenting across the inguinal ligament. J Vasc Surg. 2008;48:1255-61.

25. Delis KT, Bjarnason $H$, Wennberg PW, Rooke TW, Gloviczki P. Successful iliac vein and inferior vena cava stenting ameliorates venous claudication and improves venous outflow, calf muscle pump function, and clinical status in post-thrombotic syndrome. Ann Surg. 2007;245:130-9.

26. Hartung $O$, Barthelemy P, ArnouxD, Boufi M, Alimi YS. Management of pregnancy in women with previous left ilio-caval stenting. J Vasc Surg. 2009;50:355-9.

27. Raju S, Hollis K, Neglen P. Obstructive lesions of the inferior vena cava: clinical features and endovenous treatment. J Vasc Surg. 2006;44:820-7.

28. Néglen P, Raju S. In-stent recurrent stenosis in stents placed in the lower extremity venous outflow tract. J Vasc Surg. 2004;39:181-7.

Correspondência: Giuliano de Almeida Sandri Rua Loefgreen, 441, apto. 174 - Vila Clementino CEP 04040-030 - São Paulo (SP), Brasil E-mail:dr_sandri@hotmail.com 